\title{
Isolation and characterization of antibacterial substances produced by marine actinomycetes in the presence of seawater
}

\author{
Chiaki Imada $^{1 *}$, Naoko Koseki ${ }^{1}$, Masazumi Kamata ${ }^{2}$, \\ Takeshi Kobayashi ${ }^{1}$, and Naoko Hamada-Sato ${ }^{1}$ \\ ${ }^{1}$ Graduate School of Marine Science and Technology, Tokyo University of Marine Science and Technology, \\ 4-5-7, Konan, Minato-ku, Tokyo 108-8477, Japan \\ ${ }^{2}$ Department of Science of Aesthetics and Art, Yamano College of Aesthetics, \\ 530, Yarimizu, Hachioji, Tokyo 192-0396, Japan
}

(Received Jul. 31, 2006 / Accepted Apr. 4, 2007 / Published Jun. 14, 2007)

\begin{abstract}
Actinomycetes, which produced antibacterial substances against Gram-positive bacteria in the presence of seawater only, were isolated from marine environment and characterized. Of the $\mathbf{1 0 0}$ isolates from Otsuchi Bay in Iwate, 41 failed to produce any antibacterial substances in the absence of seawater. However, of the 41, two were found to produce antibacterial substances in the presence of seawater. Strain No. 18 exhibited higher activity than strain No. 28 and was, therefore, selected for further studies. Phylogenetic analysis and physiological characterization showed a high similarity between strain No. 18 and Micromonospora globosa. However, M. globosa JCM 3126 did not produce any antibiotics in the presence of seawater. Therefore, the effect of seawater on the growth of strain No. 18 and its production of antibacterial substances was investigated. Strain No. 18 grew in a medium prepared with artificial seawater at a concentration range of $0_{-}$ $140 \%(v / v)$. The optimal concentration of growth ranged from 10 to $30 \%(v / v)$. However, production of antibacterial substances was observed in the concentration range $60-110 \%(\mathrm{v} / \mathrm{v})$ despite poor growth. Thus, the production of antibacterial substances is seawater-dependent. The purification and characterization of the substances is now in progress.
\end{abstract}

\section{INTRODUCTION}

Natural organic compounds produced by microorganisms are an important screening target for a variety of bioactive substances. Compounds of actinomycetal origin, in particular, have been valuable in the field of bioactives. However, the rate of discovery of novel substances from microorganisms, especially from actinomycetes of terrestrial origin, has decreased recently. Most actinomycetes were believed to terrestrial; however, some strains have also been found in marine environments, though no marine actinomycetes with special characteristics have been isolated. In 2002, Mincer et al. ${ }^{1)}$ isolated actinomycetes from a marine environment, requiring seawater for growth, and these strains were designated marine actinomycetes.

Oceans cover more than $70 \%$ of the earth's surface and the organisms growing in marine environments are metabolically and physiologically different from terrestrial organisms. There are reports ${ }^{2)}$ of actinomycetes that had physiologically adapted to a marine environment. One strain, isolated from sediment of shallow seawater and designated SS-22, was identified as Streptomyces rutgersensis. Detailed observations, however, revealed that the characteristics of the strain differed from the type strain (ISP-5077) of the same species regarding the liquefaction of gelatin and tolerance to $\mathrm{NaCl}$ for growth ${ }^{3)}$. Thus, it would appear that some strains of terrestrial origin, transported to rivers through rain or irrigation and, finally, to the sea, can adapt to the marine environment. If adaptation to the environment is due to metabolic changes, the production of new bioactive compounds as metabolites may be expected. Okami et al. ${ }^{4}$ isolated a strain of Streptomyces griseus, a common soil species, from marine sediment in Sagami Bay. The strain showed no streptomycin production; however, production of new antibiotics, active against plasmodia in vivo, in a medium, low in organic nutrients and high in salt such as $\mathrm{NaCl}$, was observed. Antibiotic production was reduced with an increase in the nutrient level of the media. The chemical structure of the substance is an ionophoric polyether and is symmetric with a boron atom in the center ${ }^{5}$. Thus, a metabolite of terrestrial actinomycetes had changed over time in marine environmental conditions.

In the present paper, we report the isolation and characterization of a novel actinomycete, which produced an antibiotic in the presence of seawater but not in its absence.

\section{MATERIALS AND METHODS}

Collection of sediment samples and pretreatment before isolation of actinomycetes

Marine sediment samples were collected from Otsuchi

*Corresponding author. Phone \& FAX: +81-03-5463-0404, E-mail: imada@kaiyodai.ac.jp 
Table 1. Location of sampling stations in Otsuchi Bay

\begin{tabular}{cccc}
\hline Station & Latitude $(\mathrm{N})$ & Longitude $(\mathrm{E})$ & Depth $(\mathrm{m})$ \\
\hline 1 & $39^{\circ} 22.078^{\prime}$ & $142^{\circ} 00.664^{\prime}$ & 100.0 \\
2 & $39^{\circ} 21.900^{\prime}$ & $141^{\circ} 59.626^{\prime}$ & 80.0 \\
3 & $39^{\circ} 21.274^{\prime}$ & $141^{\circ} 58.740^{\prime}$ & 66.6 \\
4 & $39^{\circ} 20.814^{\prime}$ & $141^{\circ} 58.320^{\prime}$ & 54.0 \\
5 & $39^{\circ} 20.624^{\prime}$ & $141^{\circ} 57.808^{\prime}$ & 45.0 \\
6 & $39^{\circ} 20.378^{\prime}$ & $141^{\circ} 56.761^{\prime}$ & 42.7 \\
7 & $39^{\circ} 20.084^{\prime}$ & $141^{\circ} 54.858^{\prime}$ & 16.5 \\
8 & $39^{\circ} 23.028^{\prime}$ & $142^{\circ} 00.323^{\prime}$ & 91.2 \\
9 & $39^{\circ} 23.408^{\prime}$ & $141^{\circ} 58.688^{\prime}$ & 57.5 \\
\hline
\end{tabular}

Bay in Iwate Prefecture using a Smith-McIntyre sediment sampler on June 4th, 2001. The location and depths of these sampling stations are summarized in Table 1. The maximum depth of collection was $100 \mathrm{~m}$ and all samples were decompressed without maintaining the in situ pressure and stored at $3{ }^{\circ} \mathrm{C}$. Isolation of actinomycetes was carried out immediately upon retrieval of the samples. Each sample was dried overnight at $27^{\circ} \mathrm{C}$ to eliminate bacterial growth, followed by filtration through a $2-\mathrm{mm}$ mesh to remove large sand particles, etc.

\section{Isolation of actinomycetes from sediment samples}

Starch nitrate agar ${ }^{6)}$ and $\mathrm{HV}$ agar ${ }^{7)}$, prepared with or without seawater ${ }^{8)}$, were used for the isolation of actinomycetes in situ. Each media contained cycloheximde (50 $\mathrm{mg} / \mathrm{l})$ and nalidixic acid $(20 \mathrm{mg} / \mathrm{l})$ to prevent fungal and Gram-negative bacterial growth, respectively. The sediment samples were appropriately diluted with sterilized seawater and an aliquot of $0.1 \mathrm{ml}$ was spread on the media. After incubation for $1-3$ weeks at $27^{\circ} \mathrm{C}$, the actinomycete colonies that developed on the plates were counted and numbers were expressed in colony forming units (cfu). The colonies were picked out and purified before being stored in ISP-4 slants.

\section{Cultivation of actinomycetes for screening of antibiotic substances with or without seawater}

Actinomycetes were cultivated in disposable plastic 24well plates for the screening of antibiotic substances. Culture was carried out in about $1.5 \mathrm{ml}$ of Yeast-Malt extract medium (0.4\% Bacto-yeast extract, $1.0 \%$ Bacto-malt extract and $0.4 \%$ glucose, $\mathrm{pH} 7.3$ ) at $27^{\circ} \mathrm{C}$ for 7 days on a minishaker at $370 \mathrm{rpm}$ (type MBSS-500; B. E. Marubishi Co., Ltd, Tokyo, Japan). After growth, the culture was harvested by centrifugation at $12000 \times g$ for $15 \mathrm{~min}$ and the supernatant used for the assay of antibiotic activity by the cup assay method. The assay plates seeded with Bacillus subtilis PCI 219 using Mycin assay agar (Mikuni), Escherichia coli K-12 using Nutrient agar (Difco), and Candida albicans 3147 using Sabouraud dextrose agar were used for the antibiotic assay, respectively. Actinomycetes with no activity were further cultivated for 10 days

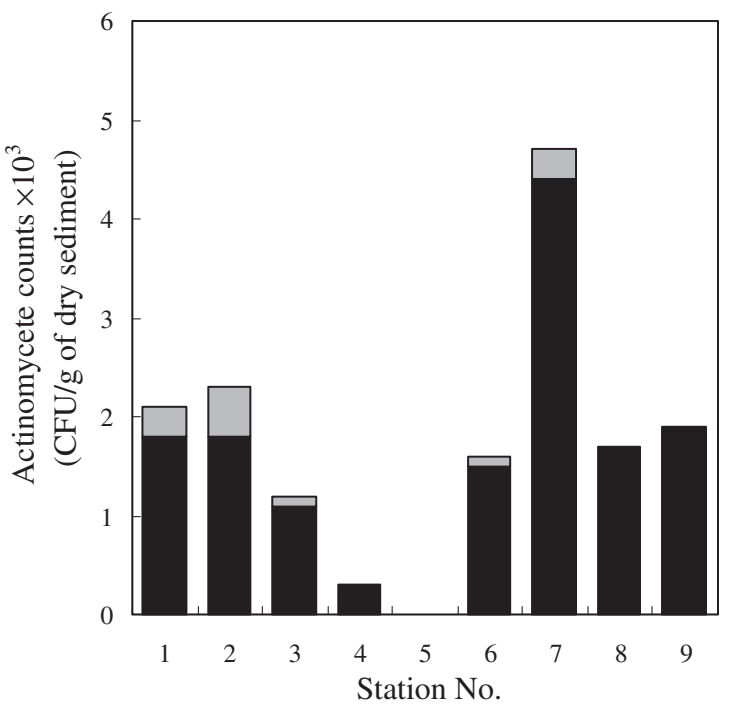

Fig. 1. Percentage of Micromonospora in the total actinomycete population at each station. $\square$ Others; $\square$ Micromonospora

in the same medium prepared with seawater and the activity was checked by the same method.

16S rDNA sequencing of the antibiotic-producing strain

The strain was inoculated into Yeast-Malt extract medium supplemented with $1 \%$ glycine and cultivated at $27^{\circ} \mathrm{C}$ for 5 days. The culture was harvested by centrifugation and the resulting precipitates were suspended in $0.2 \mathrm{ml}$ of $5 \mathrm{mM}$ EDTA (pH 8.0). Lysozyme (Wako, Osaka, Japan) was added to the suspension at a final concentration of $1 \mathrm{mg} / \mathrm{ml}$ and incubated at $37^{\circ} \mathrm{C}$ for $30 \mathrm{~min}$. After incubation, $0.02 \mathrm{ml}$ of $20 \%$ SDS $/ 0.25 \mathrm{~N} \mathrm{NaOH}$ was added and the suspension was further incubated at $55^{\circ} \mathrm{C}$ for 10 min. DNA was extracted and purified utilizing the method of Esaki et al. ${ }^{9}$. The 16S rDNA sequencing was carried out using a commercial PCR and Dye Terminator Cycle Sequencing kit (Applied Biosystems, Foster City, CA, USA). The reaction mixture was analyzed with an Applied Biosystems 310 DNA sequencer, as described previous$1 y^{10)}$. Sequence data were compiled, based on overlapping sequence data, using the GENETYX computer program (Genetyx, Tokyo, Japan). Nucleotide situation rates $\left(K_{\text {nuc }}\right.$

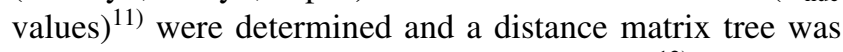
constructed by the neighbor-joining method ${ }^{12)}$ using the CLUSTAL W program.

\section{Taxonomic characteristics of the antibiotic-producing strain}

Methods and media described by the International Streptomyces Project (ISP) were used to determine most of the morphological and physiological characteristics of the strain. Inoculated media were incubated at $27^{\circ} \mathrm{C}$ for 3 weeks before observation. Detailed observations of mycelial morphology were performed under a light microscope 


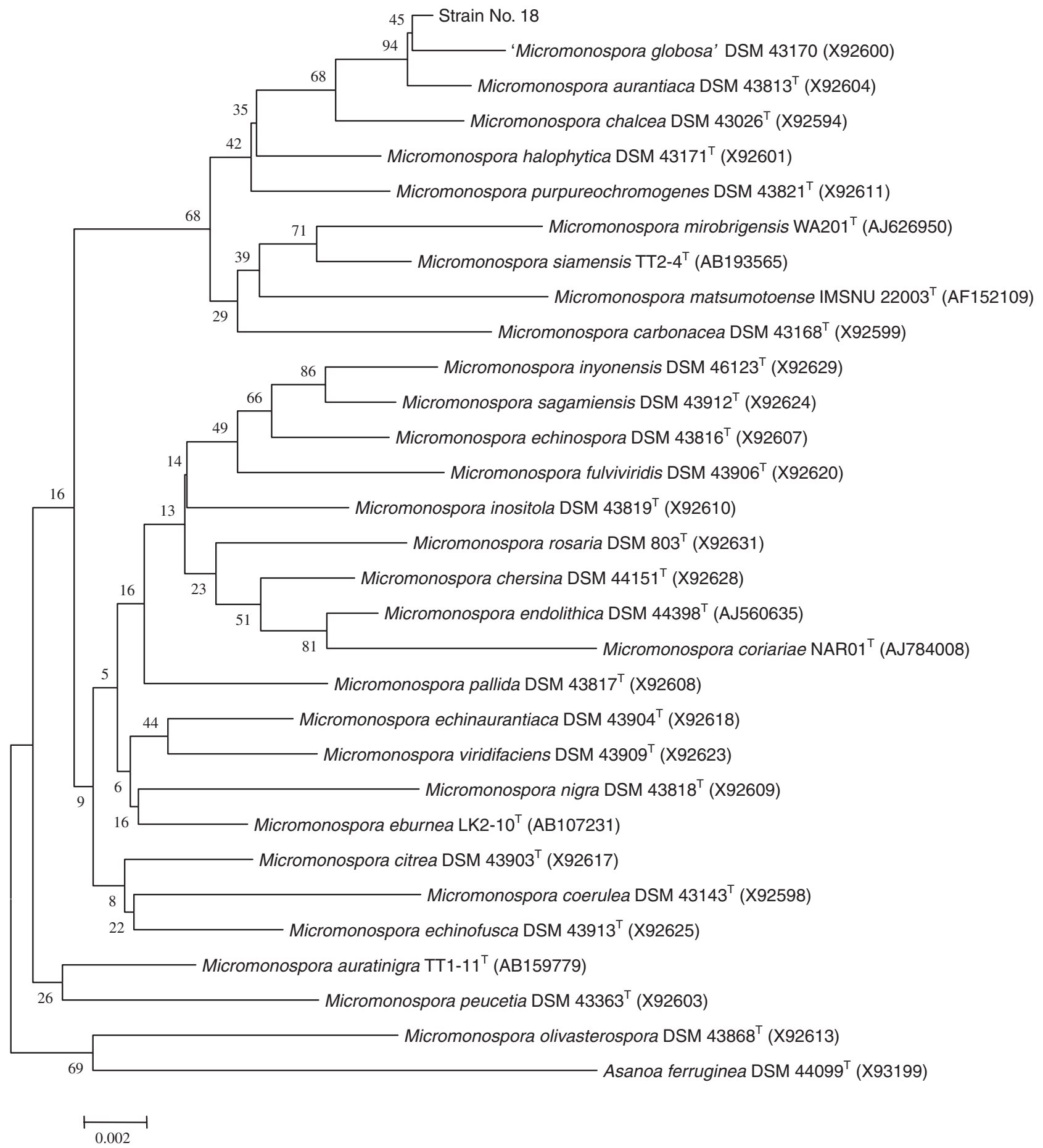

Fig. 2. Phylogenetic tree showing the position of strain No. 18 based on $16 \mathrm{~S}$ rRNA analysis. Number at nodes indicates the level (\%) of bootstrap support based on neighbor-joining analysis of 1000 resampled datasets. Only values $>50 \%$ are shown.

after the strain was incubated on ISP-2 medium. The utilization of carbon sources was examined on Pridham and Gottlieb's medium ${ }^{13)}$ containing miscellaneous carbohydrates to a final concentration of $1 \%$. $\mathrm{NaCl}$ tolerance $(0$ $13 \%)$ was tested on Yeast-Malt agar slants. Type strain Micromonospora globosa (JCM 3126) was obtained from JCM.
Time-course for growth of the strain and production of antibiotic substance with or without seawater

The time-course for growth of the strain and production of the antibiotic substances were examined using a YeastMalt extract liquid medium prepared with freshwater. Baffled 500-ml Erlenmeyer flasks, filled with $100 \mathrm{ml}$ of medium, were inoculated with the strain and precultured at 
$27^{\circ} \mathrm{C}$ for 7 days on a rotary shaker at $160 \mathrm{rpm}$ with an eccentricity of $7 \mathrm{~cm}$. Then, the strain was cultivated in $100 \mathrm{ml}$ of the same medium and antibiotic activity of the culture supernatant against B. subtilis PCI 219 was monitored. The growth of the strain was assessed by the dry weight of the mycelium from $1 \mathrm{ml}$ of the broth after drying overnight at $60^{\circ} \mathrm{C}$.

To investigate the effect of seawater concentration on the growth and production of antibiotic substances, a YeastMalt extract liquid medium prepared with various concentrations $(0-200 \%, \mathrm{v} / \mathrm{v})$ of artificial seawater were used. Cultivation was carried out at $27^{\circ} \mathrm{C}$ for 10 days and the growth of the strain and antibiotic activity measured.

To find the essential compound for the production of antibiotics, every cation contained in seawater was eliminated and the growth of the strain and production of antibiotic substances were determined as described above.

\section{RESULTS AND DISCUSSION}

\section{Distribution of actinomycetes in marine sediment}

Figure 1 shows the percentage of genus Micromonospora in the total actinomycete population at each sampling station. The number of actinomycete colonies is much higher in medium without than with seawater from all stations, except for Stn-5 (data not shown). The highest actinomycetes count was observed in Stn-7 due to terrestrial influences. No actinomycete colonies were found in Stn-5, since the bottom substrate contains sand particles. The percentage of genus Micromonospora, judged by the eye for morphological characteristics, in the total actinomycete population is high for all stations. These results agree with results for the population of Micromonospora colonies in seawater media ${ }^{14,15)}$. The population of Micromonospora in the bay is much higher compared with other sea areas.

\section{Cultivation of actinomycetes for screening of antibiotic substances with or without seawater}

Of 100 strains from various sea areas tested for the production of antibiotic substances without seawater medium, 59 strains were found to produce substances, whereas 41 were non-producers. However, of the 41 strains, two were found to produce substances with seawater. Among these, the strain isolated from Stn-1 (100 m depth) and designated No. 18, exhibited higher activity and was selected for further studies.

\section{Characterization and identification of strain No. 18}

Phylogenetic analysis by 16 S rDNA sequencing (1500 bp) showed a high similarity between strain No. 18 and Micromonospora globosa (similarity: 99.06\%) (Fig. 2). The strain showed close similarity with $M$. aurantiaca (98.94\%). The colony of strain No. 18 was yellow/orange in color with a waxy and leathery, tough texture, showing good growth with the formation of a black spore layer on

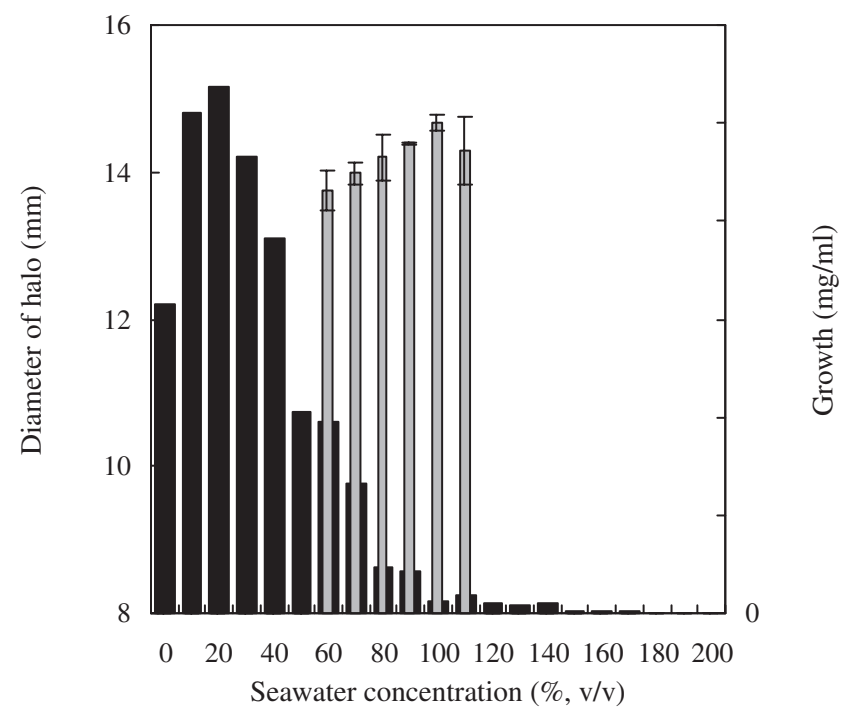

Fig. 3. Effect of seawater concentration on the growth and production of antimicrobial substances. G Growth, $\square$ Antimicrobial activity

the ISP-4 agar medium. The strain was Gram-positive, did not make aerial mycelium, did not produce sporangia and produced nonmotile spores that were borne individually on branched hyphae. The colonies become darkened during sporulation with spores produced singly on the colony surface. Vegetative hyphae are finely branched and do not fragment. These morphological characteristics permit the strain to be classed in the genus Micromonospora. It was tolerant of $\mathrm{NaCl}$ concentrations up to $4 \%$ but did not require the presence of $\mathrm{NaCl}$ or seawater for growth. The strain produced a red color pigment in the presence of seawater $(70-110 \%, \mathrm{v} / \mathrm{v})$ in both liquid and agar media.

Effect of seawater concentration and various cations in seawater on growth and production of antibiotic substances

Slow growth was observed after inoculation of the strain and maximal growth was obtained after 14 days of cultivation. Antibiotic activity was observed after 6 days of cultivation, reaching a maximum in 7 days, with a decline in activity thereafter (data not shown).

Figure 3 illustrates the effect of seawater concentration on the growth and production of antibiotic substances. The strain grew in seawater at the range $0-140 \%(\mathrm{v} / \mathrm{v})$. The optimal concentration ranged from 10 to $30 \%(\mathrm{v} / \mathrm{v})$. However, production of antibacterial substances was observed in the concentration range $60-110 \%(\mathrm{v} / \mathrm{v})$ despite poor growth. Thus, the production of antibiotic substances is seawater-dependent. M. globosa is known to produce an antibiotic called deoxydynemicin $\mathrm{A}$ in the absence of seawater as the strain was isolated from a soil sample ${ }^{16)}$. It is interesting that $M$. globosa, living in a marine environment, developed this metabolism. 


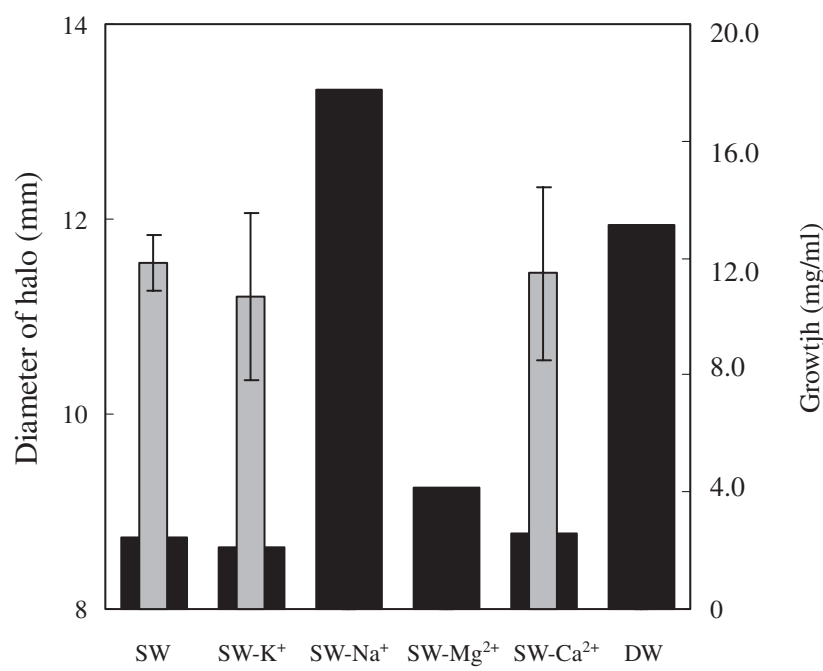

Fig. 4. Growth of strain No. 18 and production of antibiotic substance after removal of each salt from artificial seawater.

- Growth, $\square$ Antimicrobial activity SW, Seawater; DW, Deionized water

As shown in Fig. 4, good growth was observed in the medium without $\mathrm{Na}^{+}$or distilled water, whereas no production of antibiotics was observed. Thus, $\mathrm{Na}^{+}$is essential for production. Streptomyces aureofaciens is known to have no $\mathrm{NaCl}$ tolerance for growth; however, the strain grows gradually under increased concentration of $\mathrm{NaCl}$ after repeated subculturing on the agar medium. UV spectra of the culture supernatant of the strain before and after adaptation to increased concentrations of $\mathrm{NaCl}$ are considerably different, suggesting that the metabolite might be changed after adaptation to $\mathrm{NaCl}^{17)}$. It appears that strain No. 18 had the same mechanism of antibiotic production.

Further studies on the purification of the antibiotic substance and elucidation of its production pathways are underway.

\section{ACKNOWLEDGEMENT}

The authors acknowledge Dr. Y. Igarashi of Toyama Prefectural University for his valuable comments.

\section{REFERENCES}

1) Mincer, T. L.; P. R. Jensen, C. A. Kauffman \& W. Fenical: Widespread and persistent populations of a major new marine actinomycete taxon in ocean sediments. Appl.
Environ. Microbiol. 68: 5005-5011, 2002

2) Takizawa, M.; R. R. Colwell \& R. T. Hill: Isolation and diversity of actinomycetes in the Chasapeake Bay. Appl. Environ. Microbiol. 59: 997-1002, 1993

3) Arai, T.: Actinomycetes, the Boundary Microorganisms. Toppan, Tokyo, 123, 1976

4) Okami, Y.; T. Okazaki, T. Kitahara, \& H. Umezawa: Studies on marine microorganisms. V. A new antibiotic, aplasmomycin, produced by a streptomycete isolated from shallow sea mud. J. Antibiot. 29: 1019-1025, 1976

5) Nakamura, H.; Y. Iitaka, T. Kitahara \& T. Okazaki: Structure of aplasmomycin. J. Antibiot. 30: 714-719, 1977

6) Ghanem, N. B.; S. A. Sabry, Z. M. Ei-sherif \& G. A. A. El-Ela: Enumeration of marine actinomycetes from seawater and sediments in Alexandria. J. Gen. Microbiol. 46: 105111,2000

7) Hayakawa, M. \& H. Nonomura: New actinomycete selective isolation medium $\mathrm{HV}$ agar. (In Selective isolation of terrestrial actinomycete) pp. 4-25 1993 (in Japanese)

8) Imada, C.; K. Hotta \& Y. Okami: A novel marine Bacillus with multiple amino acid analog resistance and selenomethionine-dependent antibiotic productivity. J. Mar. Biotechnol 6: 189-192, 1998

9) Ezaki, T.; Y. Hashimoto, H. Yamamoto, M. L. Lucida, S. Liu, S. Kusunoki, K. Asano \& E. Yabuuchi: Evaluation of the microplate hybridiation method for rapid identification of Legionella species. Eur. J. Cli. Microbiol. Infec. Dis. 9: 213217, 1990

10) Kobayashi, T.; B. Kimura \& T. Fujii: Haloanaerobium fermentans sp. nov. a strictly anaerobic fermentative halophile isolated from fermented puffer fish ovaries. Int. J. Syt. Evol. Bacteriol. 50: 1621-1627, 2000

11) Kimura, M.: A simple method for estimating evolutionary rates of base substitutions through comparative studies of nucleotide sequences. J. Mol. Evol. 16: 111-120, 1980

12) Saitou, N. \& M. Nei: The neighbor-joining method: a new method for reconstructing phylogenetic trees. Mol. Biol. Evol. 4: 406-425, 1987

13) Pridham, T. G. \& D. Gottlieb: The utilization of carbon compounds by some actinomycetes as an aid for species determination. J. Bacteriol. 56: 107-114, 1948

14) Wakisaka, Y.; Y. Kawamura, Y. Yasuda, K. Koizumi \& Y. Nishimoto: A selective isolation procedure for Micromonospora. J. Antibiot. 35: 822-836, 1982

15) Jensen, P. R.; R. Dwight \& W. Fenical: Distribution of actinomycetes in nearshore tropical marine sediments. Appl. Environ. Microbiol. 58: 1102-1108, 1991

16) Shiomi, K.; H. Iinuma, H. Naganawa, M. Hamada, S. Hattori, H. Nakamura \& T. Takeuchi: New antibiotic produced by Micromonospora globosa. J. Antibiot. 43: 1000-1005, 1990

17) Okazaki, T. \& Y. Okami: Actinomycetes tolerant to increased $\mathrm{NaCl}$ concentration and their metabolites. $J$. Ferment. Technol. 53: 833-840, 1975 